\section{A FORTRAN IV program for the measurement and statistical analysis of higher order units of subjective organization in multitrial free recall learning}

\author{
ERIC T. ROACH \\ Department of Public Health \\ Inkster, Michigan 48141 \\ and \\ ROBERT R. WALSH \\ Department of Corrections \\ Jackson, Michigan 49201
}

The program described here was written to facilitate the measurement and statistical analysis of higher order subjective organization data obtained from multitrial free recall experiments. There has been increasing interest in the investigation of subjective organization and the structure of recalled words lacking strong or obvious interitem relationships. Much of the research, however, has been hindered by inadequacies associated with the most frequently employed measures of subjective organization, namely, the ratio measure of Tulving (1962) and the intertrial repetition (ITR) deviation index of Bousfield and Bousfield (1966).

A frequent criticism of these measures (Postman, 1972; Shuell, 1969) concerns their reliance on the fixed trial to trial consistency of response orderings in determining subjective organization, such that only pairwise item dependencies are taken into account. Thus, for a recall sequence containing groupings of two or more items to be scored as a single unit, each of the constituents must be recalled in the same sequential order on Trial $t+1$ as on Trial $t$. As a consequence, many investigations employing these measures are, in practice, ultimately reduced to determining the presence or absence of item pairs connected as a unit solely as simple forward associations. The possibility of higher order subjective units occurring, varying in size and internal ordering sequence, is excluded from analysis.

The serious limitations imposed can be easily illustrated, for example, by considering a stimulus list consisting of 12 items, A-L. Assume that on Trial $t$, seven of the items are recalled in the sequence BCDEAJK, followed by recall on Trial $t+1$ of CBDELHKJ. Both the Tulving and the Bousfield measures would only score the two-item unit DE as an observed ITR. Note, however, that the item pairs $\mathrm{CB}$ and $\mathrm{KJ}$ were recalled together on Trial $\mathrm{t}+1$ in reverse sequential order. The possibility of bidirectional ordering variation was considered by Gorfein, Blair, and Rowland (1968), who modified the Bousfield formula to accommodate this alternative grouping.
If the Gorfein et al. modification is incorporated, there are three observed ITR units of two items each occurring in the recall of the above trial pair.

The bidirectional fixed sequence consideration does not, however, exhaust the possibility of additional organization being present in the above recall example. If we consider that the first four items common to both trials are identical, then it seems possible that they, too, may represent a single recall unit. The sequence of CBDE represents 1 of the 4 ! possible permutations of items BCDE. While they do not occur in a fixed pairwise sequence, they are recalled as an intact grouping composed internally of the same elements. Pellegrino (1971) has incorporated this notion, suggesting that an additional criterion, that of unordered consistency, be employed in examining free recall protoeols for subjective organization.

The technique suggested by Pellegrino allows determination of the amount of organization present under each criterion: unidirectional, bidirectional, and unordered. Within each criterion, the recall sequence is examined successively for units varying in size from 2 to $M$, where $M=$ the number of items recalled on Trial $t$. The general measure provides a separate ARC (adjusted ratio of clustering) score for each unit size and ordering criterion based on the ratio of observedexpected ITRs to the maximum possible-expected ITRs. Further details on the procedure, along with the formulae, can be found in Pellegrino (1971).

\section{Program SOFR}

This program calculates and prints the individual ARC (adjusted ratio of clustering) scores for each subject, along with values for the various parameters needed to calculate the expectation of an intertrial repetition by each of the recall unit ordering criteria (unidirectional, bidirectional, unordered). SOFR is a further correction (cf. Walsh \& Roach, 1974) and extension of the program presented by Pellegrino (1972), and it contains optional subroutines for a three-way analysis of variance, as well as for analysis of interaction effects (both are discussed later).

The first card of the data deck consists of parameters delineating the architecture of the split-plot analysis of variance (as discussed in the section on subroutine SPLIT). The data on this card are five two-digit numbers, designated IN, IP, IQ, IZ, and IT. IN is the number of subjects per condition cell; IP is the number of plots; IQ is the number of trials per subject; IZ is the number of conditions to be studied in each plot; and IT is the total number of subjects in the experiment. IT is redundant and is used solely as an internal programming consistency check. An example of this card for an experiment involving 72 subjects (IT), divided into two plots (IP), with four condition vari- 
ations (IZ), 9 subjects per condition cell (IN), and nine trials per subject (IQ), would be:

\section{2}

If the total number of subjects exceeds 99, Card 20 must be modified from:

\section{FORMAT(5I2)}

to:

$$
50 \text { FORMAT }(4 \mathrm{I} 2, \mathrm{I} 3)
$$

The second card of the data input deck is designated the general parameter card, and contains two values: NS, or number of subjects (identical to IT on the previous card), and MS, the number of items on the recall list. NS occupies the first three digits of the second data input card, and MS is in the fourth and fifth columns. Thus, for the example in the preceding paragraph, with 11 items on the recall list, the second data card in the deck would be:

\section{1}

The actual input data consist of the recall position of the stimulus word, entered as a two-digit numeric for each trial. If the word was not recalled on the trial, then a 00 would be entered. Each pair of columns on the Hollerith card, beginning with Column 1, corresponds to a single trial. Each subject will have a data card for each item in the stimulus list. Thus, for example, for a given list of 11 words, A-K, presented over nine trials, where Item $A$ was recalled six times in the following sequence:

\section{Trial}

\begin{tabular}{|c|c|}
\hline & 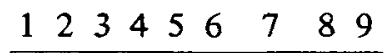 \\
\hline Recall Position & $\begin{array}{lllllllll}0 & 0 & 3 & 0 & 1 & 7 & 11 & 10 & 6\end{array}$ \\
\hline
\end{tabular}

the data would be entered in the first 18 columns of the card as:

\section{6}

Preceding each block of individual item data cards will be a subject parameter and identification card. The first two columns of this card contain the number of trials, the third and fourth columns contain the size of the largest subjective organization unit the experimenter wants to analyze (range 2-10), and the fifth, sixth, and seventh columns contain the subject identification number. Thus, for a nine-trial presentation, in which the investigator is interested in examining units no larger than Size 5, the card for Subject 1 would be:
As written, SOFR has a maximum capacity for analyzing recall of a 40-item list over 20 trials, with 72 subjects. To modify the last capacity upward for larger experiments, the user should change Columns 22 and 23 of the following two cards (Statement 18 SOFR, Appendix A):

\section{COMMON/A/SAVE $(72,30,4)$}

and (Statement 10 in SPLIT, Appendix A):

\section{COMMON/A/SAVE(72,30,4)/B/MATRIX(16,20,20)}

to reflect the number of subjects utilized. Thus, for example, if the investigator desired an analysis for a free recall experiment containing 144 subjects, the above statements would be changed in the following manner to reserve more common core:

\section{COMMON/A/SAVE $(144,30,4)$}

and

\section{COMMON/A/SAVE(144,30,4)/B/MATRIX(16,20,20)}

Conversely, the statements can similarly be modified downward for smaller experiments and for economy of core when the user's computer has a limited core capacity.

The complete output for SOFR includes the number of observed word repetitions, expected repetitions, maximum possible repetitions, deviation values, and ARC scores for each trial pair by ordering criteria and size of subjective organization unit examined. Also printed are the values of $R, M, N$, and mean ARC scores averaged over trial pairs. This output is described below.

Observed repetitions. The first item printed following the subject identification number consists of the actual number of observed intertrial repetitions, presented in tabular form. The rows in the table contain each trial pair (e.g., "Trial 2" refers to Trials 1 and 2; "Trial 3" refers to Trials 2 and 3, etc.) with the accompanying ordering criteria. The column heads refer to the size of the unit examined. The numerical entry is the actual number of repetitions occurring in the recall for that trial pair, ordering criterion, and size.

Expected repetitions. This is the second item printed by SOFR, and its tabular layout is the same as in the case of the observed repetitions. The numeric value of the expectation is displayed by unit size, ordering criterion, and trial pair. The value is determined according to the following formula given by Pellegrino (1971):

$$
E(\text { ITR })=\frac{(N-X+1) !(A)(M-X+1-R)}{N !},
$$


where $M=$ the total number of items recalled on Trial $t$; $\mathrm{N}=$ total recall on Trial $\mathrm{t}+1 ; \mathrm{R}=$ the number of units of Size $X$ recalled on Trial $t$ containing one or more words not recalled on Trial $t+1$; and, $A=a$ variable parameter whose value is dependent on the ordering criterion under consideration.

Maximum possible repetitions. This is the third table produced by the program and it contains row values corresponding to trial pairs, while the columns contain the unit size. The maximum possible number of observed repetitions is determined by the formula recommended by Pellegrino (1971):

$$
\operatorname{MAX}(I T R)=M-X+1-R .
$$

Deviation score. This is the fourth item printed, and its tabular format is the same in row and column designations as that for the observed repetitions. The deviation score for each ordering criterion by trial pair and unit size is presented. This value is simply the observed minus the expected number of intertrial repetitions.

ARC scores. This is the fifth table printed, and its format is similar to that for the observed repetitions. The ARC value, by ordering criterion, trial pair, and unit size is determined by the ratio of observed minus expected repetitions. Here, total or perfect organization is represented by a value of 1.0 , while chance organization is set at 0 .

$R$ values for all trials. This is the sixth table printed. The rows identify trial pairs, while the columns refer to the unit size under consideration. The numerical entries are the number of units of that specified size recalled on Trial $t$ that contain one or more items not recalled on Trial $t+1$.

Mean ARC scores. This is the seventh item printed, and it contains the average subjective organization values taken over trial pairs, listed by ordering criterion (rows) and by unit size (columns).

$\mathbf{M}$ and $\mathbf{N}$ values. This is the last item printed by SOFR. The rows correspond to individual trials (not trial pairs). The first column (unlabeled) contains the value of $M$ (the number of items recalled on Trial $t$ ); the second column contains the $\mathrm{N}$ value (the number of items recalled on Trial $t+1)$. This data ends the SOFR output for the indicated subject, and the output for the next subject begins.

Finally, it is possible to completely suppress the printing of SOFR, while continuing to execute the program. To suppress, add the following cards to the source deck at the designated locations:

\section{Add Between Statements}

$\begin{array}{lr}\text { GO TO } 2000 & 25 \text { and } 26 \\ \text { 2000 CONTINUE } & 29 \text { and } 30 \\ \text { GO TO } 2001 & 169 \text { and } 170\end{array}$
2001 CONTINUE
GO TO 2002
236 and 237
2002 CONTINUE
243 and 244
259 and 260

Inclusion of the above statements in the indicated positions saves, but does not print, the SOFR output for use in the statistical subroutines to be described below.

\section{Subroutine SPLIT}

This subroutine performs a three-way univariate analysis of variance on the subjective organization scores calculated by SOFR. It is written for a splitplot factorial experimental design containing two between-blocks treatments and one repeated or withinblocks factor corresponding to trials. The manual computations for this design, designated as a Type SPF-pr.q design, are described in detail by Kirk (1968), whose notation will be utilized below for ease of crossreference.

For the analysis, the subroutine calculates the error terms in accordance with the mixed effects or Model III analysis of variance where Factors $A, B$, and $C$ are treated as fixed effects, and subjects are treated as a random effect. Following Kirk, the two betweenblocks factors will be designated as consisting of $p$ levels of $a_{i}$ and $r$ levels of $c_{k}$, and the within-factor as $q$ levels of $b_{j}$. In its present form, subroutine SPLIT will accommodate values of $q$ up through 20 . The capacity of the between-blocks factors together is limited to 16 levels (i.e., p x r $\leqslant 16$ ). For more complex experiments, the latter capacity can easily be increased upward to a maximum combined $\mathrm{px} r$ value of 99 . To modify this capacity, simply change the values in Columns 39 and 40 of Statement 10 in SPLIT, and Columns 23 and 24 of Statement 8 in subroutine SPLITAI to reflect the desired value. For example, if $p=12$ and $r=8$, change:

COMMON/A/SAVE(72,30,4)/B/MATRIX(16,20,20)

to read:

$$
\text { COMMON/A/SAVE(72,30,4)/B/MATRIX(96,20,20) }
$$

and change:

$$
\text { COMMON/B/MATRIX }(16,20,20)
$$

to read:

\section{COMMON/B/MATRIX(96,20,20).}

Increasing the size of the matrices in the common blocks may increase the required core capacity to the point that it may exceed the capacity of the computer. If this situation obtains, the user should consult an 
experienced programmer for assistance in using auxiliary memory for temporary storage of data and calculational matrices.

This subroutine does not require additional data card preparation, as it utilizes for its input the multiple ARC scores obtained from SOFR. In its present version, SPLIT calls up a second subroutine that performs a simple effects analysis on the main effects and interactions (to be discussed below), and the output consists of identified sums of squares for all sources of variation in the design, in addition to the overall $F$ test values. To obtain only the output of subroutine SPLIT, simply remove the following card (Statement 53 of SPLIT) from the source deck:

\section{CALL SPLITAI (IP, IQ, IR, IN, NX, NT, IT)}

Deletion of the above card will limit the statistical output to the following two categories:

ARC score input values. These are the data values used in the analysis of variance arranged in a matrix of Dimension $a_{i} c_{k} \times b_{j}-1$, where the rows correspond to $N$ (the total number of subjects) listed sequentially by Treatment Combination $\mathrm{a}_{\mathrm{i}} \mathrm{c}_{\mathrm{k}}$, and columns correspond to $b_{j}-1$, the number of levels of the repeated factor (trials) minus one (trial pairs). Each cell entry is that particular subject's ARC score value for the trial pair.

Analysis of variance table. This output is printed in the form of a standard analysis of variance source table, with the row designations identifying the sources of variation, while the column heads label the sums of squares (SS), degrees of freedom (DF), mean squares (MS), and the $F$ ratio value.

The above output is printed sequentially for the ARC score values by ordering criterion and unit size, with each sequence appropriately identified (e.g., "BEGIN UNIDIRECTIONAL SIZE 2").

\section{Subroutine SPLITAI}

This subroutine is optional and may be of interest to the investigator employing a three-way analysis of variance design in which there are multiple levels of treatment components and there is significant interaction present. SPLITAI provides a labeled sums of squares calculation for the simple main effects components of all two-way interactions. In addition, the sums of squares for both the simple main effects and simple interactive components of the three-way interaction are provided. As currently written, SPLITAI cannot be used independently of subroutine SPLIT. Output is printed sequentially for each unit of subjective organization examined, by ordering criterion and unit size. Since SPLITAI is dependent upon SPLIT, the SPLIT output will always be printed when SPLITAI is executed. The combined output sequence is described below.
ARC score input values. (Described previously under Subroutine SPLIT.)

Sums of squares for all interaction components. This output is arranged in a single column with each row labeled according to the source of the component. Because of the repeated measures factor, some components will require use of different error terms in determining the appropriate value of $F$. For components involving only nonrepeated factors (e.g., $A$ at each level $c_{k}$ ), the investigator should use the mean square for subjects within groups. When the repeated factor (either singularly or in interaction) is being considered over levels of a nonrepeated factor (e.g., B x C at each level $a_{i}$ ), then the correct error term would be the mean square for Factor B by subjects within groups. Finally, when a nonrepeated factor (either singularly or in interaction) is being considered over levels that contain a nonrepeated factor (e.g., $A$ at each level $b_{j} c_{k}$ ), a pooled error term should be utilized. The correct pooled error term for this design, as given by Kirk, is determined by multiplying the mean square for $B$ by subjects within groups times $q-1$ times the mean square for subjects within groups, divided by $\mathrm{q}$.

The subroutine generates the sums of squares values for the interactive components in the design in the sequence indicated below:

Sums of squares for Factor $A$ at Levels $b_{1}-b_{j}$.

Sums of squares for Factor $B$ at Levels $a_{1}-a_{i}$.

Sums of squares for Factor $A$ at Levels $c_{1}-c_{k}$.

Sums of squares for Factor $C$ at Levels $a_{1}-a_{i}$.

Sums of squares for Factor B at Levels $c_{1}-c_{k}$.

Sums of squares for Factor $C$ at Levels $b_{1}-b_{j}$.

Sums of squares for Factor $A$ at Levels $b_{1} c_{1}-b_{j} c_{k}$.

Sums of squares for Factor $B$ at Levels $a_{1} c_{1}-a_{j} c_{k}$.

Sums of squares for Factor $C$ at Levels $a_{1} b_{1}-a_{i} b_{j}$.

Sums of squares for Factor $A \times B$ at Levels $c_{1}-c_{k}$.

Sums of squares for Factor $A \times C$ at Levels $b_{1} \cdot b_{j}$.

Sums of squares for Factor $B \times C$ at Levels $a_{1}-a_{i}$.

Analysis of variance table. (Described previously under Subroutine SPLIT.)

\section{REFERENCES}

Bousfield, A. K., \& Bousfield, W. A. Measurement of clustering and of sequential constancies in repeated free recall. Psychological Reports, 1966, 19, 935-942.

Gorfein, D. A., Blair, C., \& Rowland, C. The generality of free recall: I. Subjective organization as an ability factor. Psychonomic Science, 1968, 11, 279-280.

KIRK. R. E. Experimental design: Procedures for the behavioral sciences. Belmont: Brooks/Cole, 1968.

Pellegrino, J. W. A general measure of organization in free recall for variable unit size and internal sequential consistency. Behavior Research Methods \& Instrumentation, 1971, 3, 241-246.

Pellegrino, J. W. A Fortran IV program for analyzing higher order subjective organization units in free recall learning. Behavior Research Methods \& Instrumentation, 1972, 4, 215-217.

Postman. L. A pragmatic view of organization theory. In E. 
Tulving \& W. Donaldson (Eds.), Organization of memory. New York: Academic Press, 1972. Pp. 3-48.

Shuell, T. J. Clustering and organization in free recall. Psychological Bulletin, 1969, 72, 353-374.

Tulving, E. Subjective organization in the free recall of unrelated words. Psychological Review, 1962, 69, 344-354.

WALsh, R. R., \& RoAch, E. T. A note on Pellegrino's (1972) program for analyzing higher-order subjective organization units in free recall. Behavior Research Methods \& Instrumentation, 1974, 6, 565.

Appendix A: Program Listings for SOFR, SPLIT and SPLITAI

PROGRAM SOFR (INPUT, OUTPUT, TAPE5= INPUT)

TEST RUN DATE 13/05/74, DATA FOR SUBJECTS 1 - 8 (2ND LIST. ONLY.)

NS=NUMBER OF SUBJECTS

NT= NUMBER OF TRIALS FOR THE SUBJECT TO BE EXAMINED

MS=NUMBER OF ITEMS ON LIST

NX=SIZE OF THE LARGEST ORGANIZATIONAL UNIT TO BE EXAMINED

NSS=SUBJECT IDENTIFICATION NUMBER

MA $(I, J)$ CONTAINS THE DATA ARRANGED IN MATRIX FORM. I RUNS FROM 1 TO MS. J RUNS FROM 1 TO NT.

15

DIMENSION $\operatorname{EXP}(3,20,9), \operatorname{DEV}(3,20,9), \operatorname{ARC}(3,20,9), E(3)$

DIMENSION NN(40), MiN $(40,20), J T R(3), \operatorname{ITR}(3,20,9), \operatorname{MAX}(20,9), \operatorname{IX}(10), A R C$

11(3), MATRIX $(8,9,9)$

DIMENSION IR $(20,10)$

REAL MEAN $(3,10)$

COMON/N/SAVE $(72,30,4)$

2050 FORMAT(5I2)

READ 50,IN,IP,IQ,IZ, IT

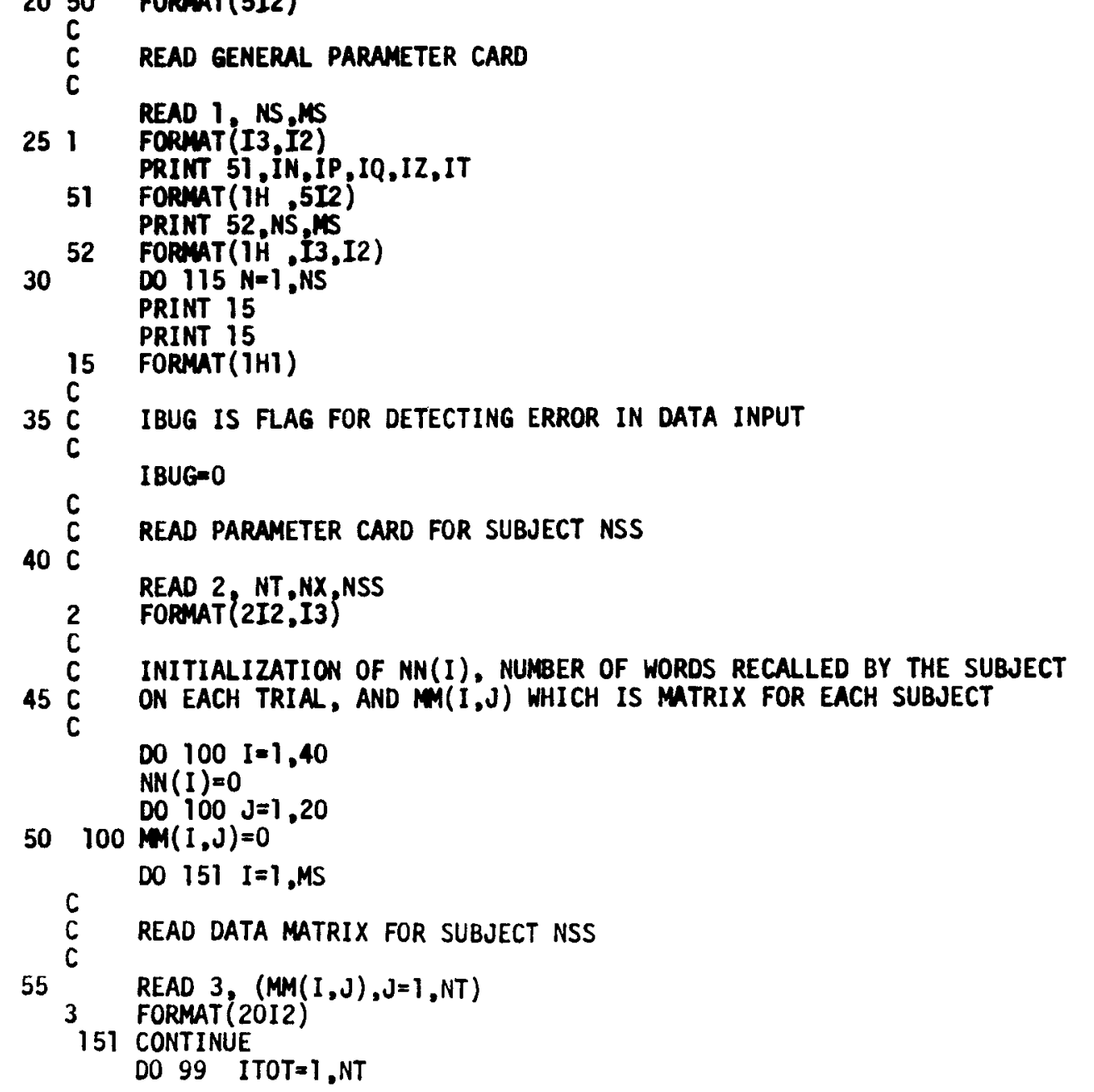




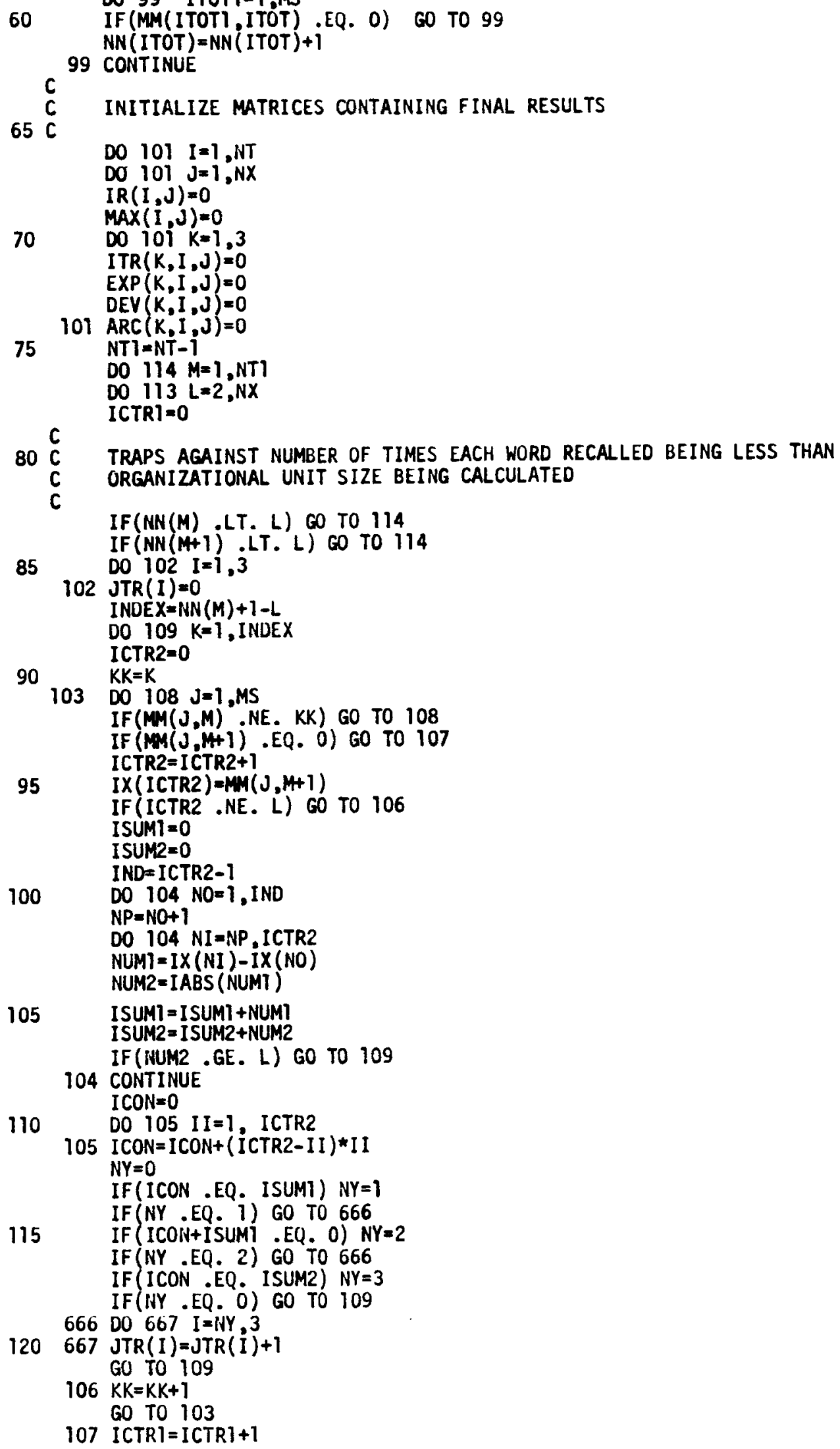

105

ISUMI $=$ ISUMI +NUMI

ISUM2 = I SUM2+ NUM2

666 DO $667 \quad I=N Y, 3$

$667 \operatorname{JTR}(I)=\operatorname{JTR}(I)+1$

GO TO 109

$106 \mathrm{KK}=\mathrm{KK}+1$

GO TO 103

$107[C T R]=[C T R]+1$ 


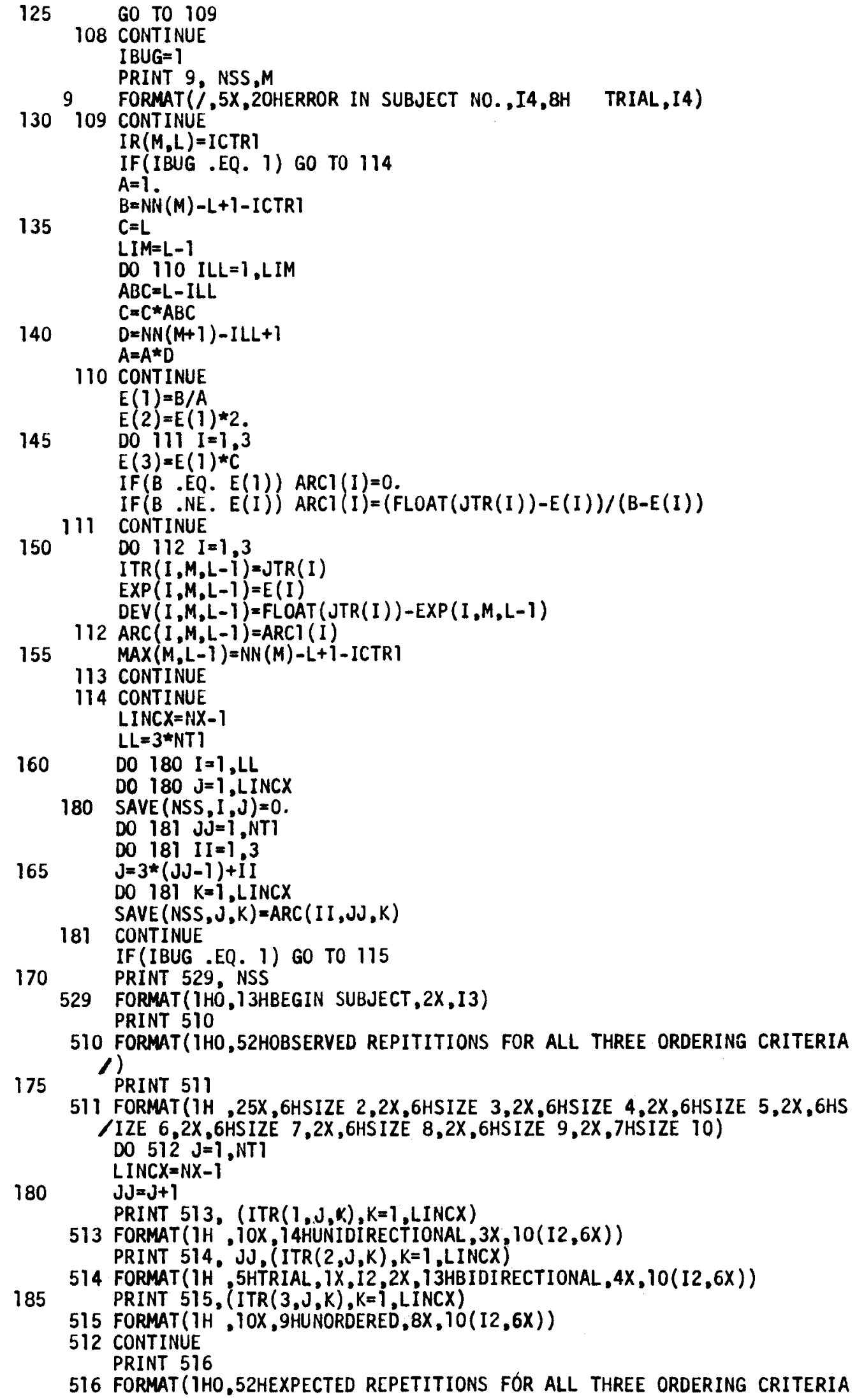




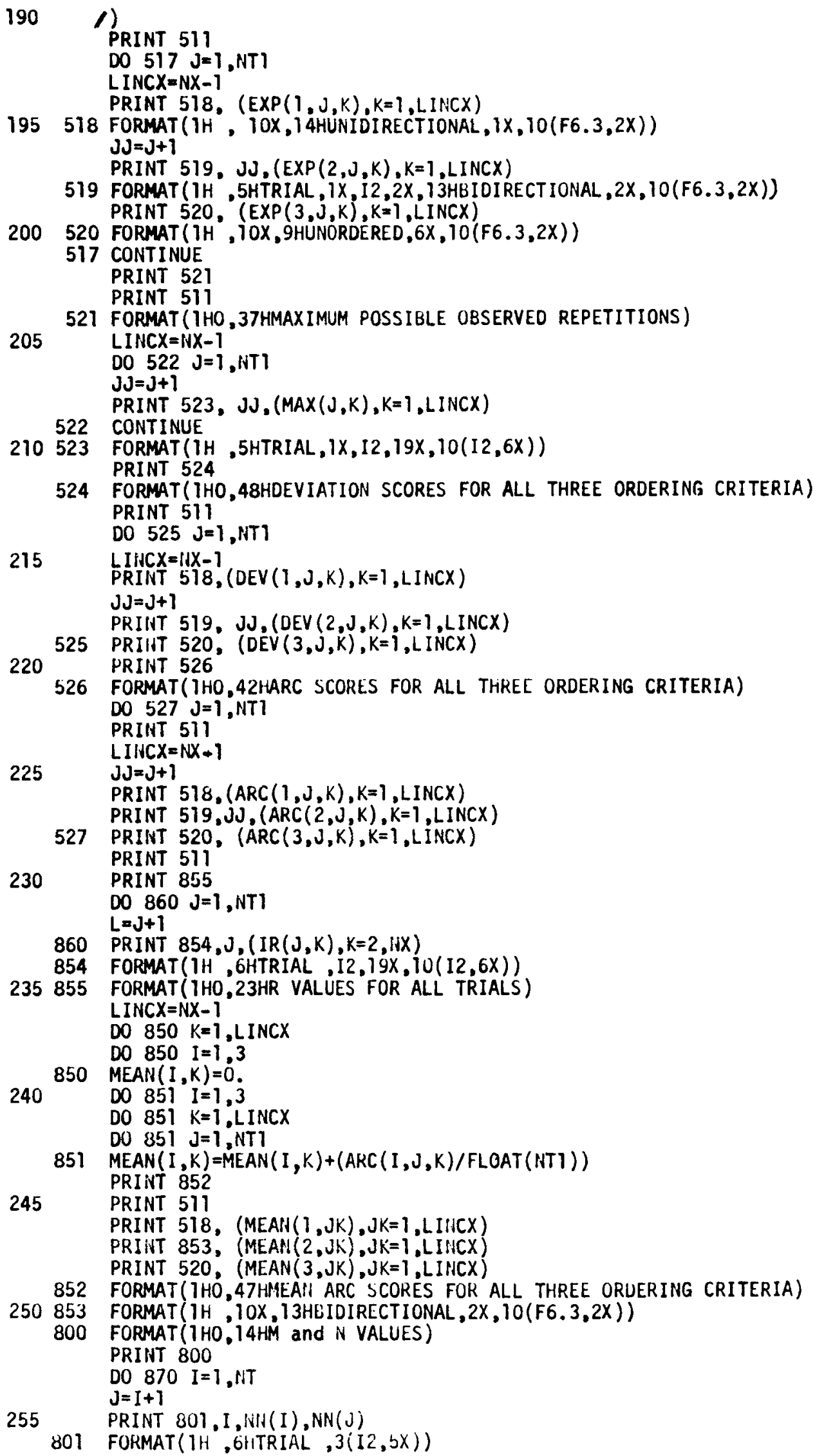




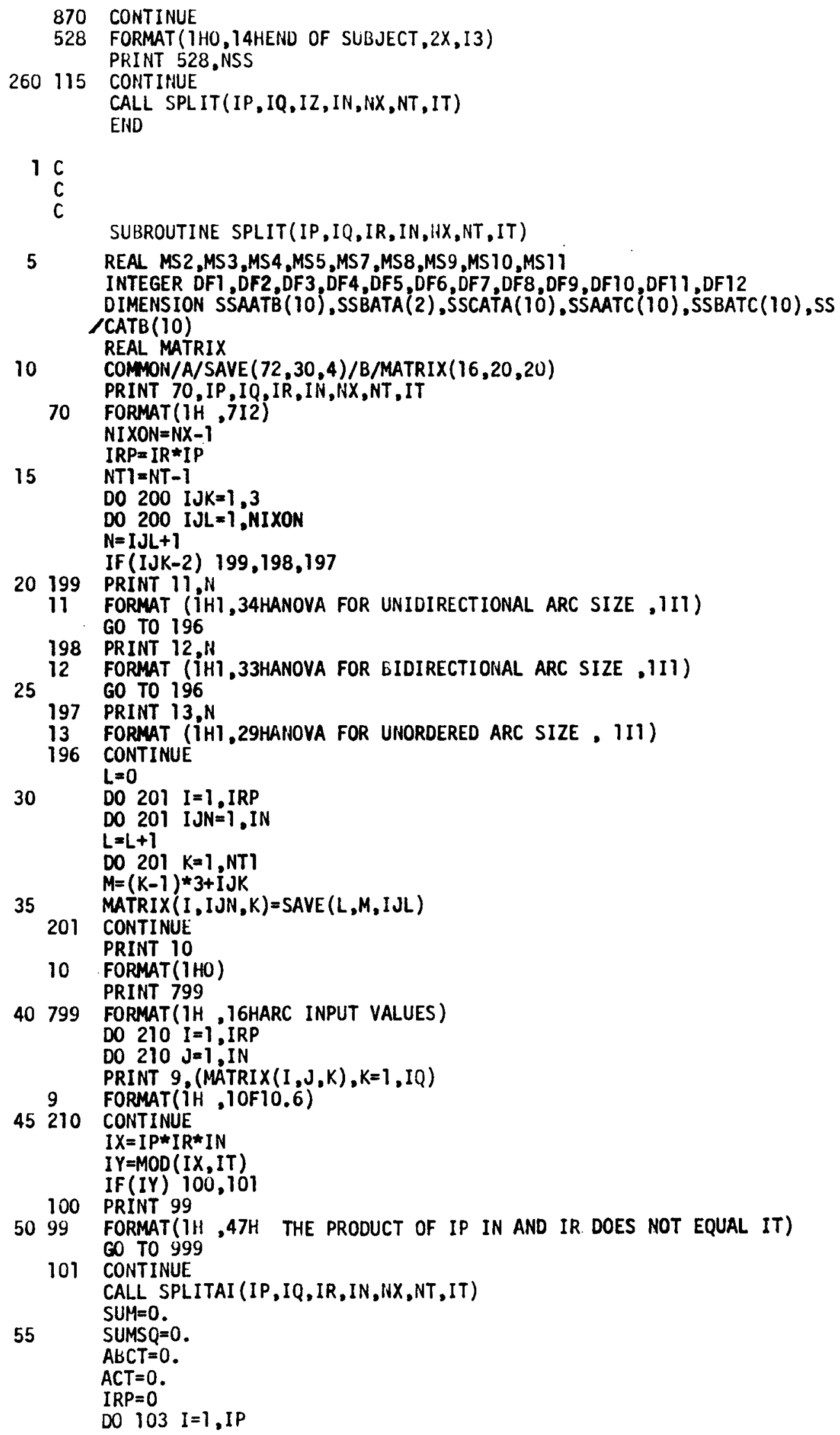




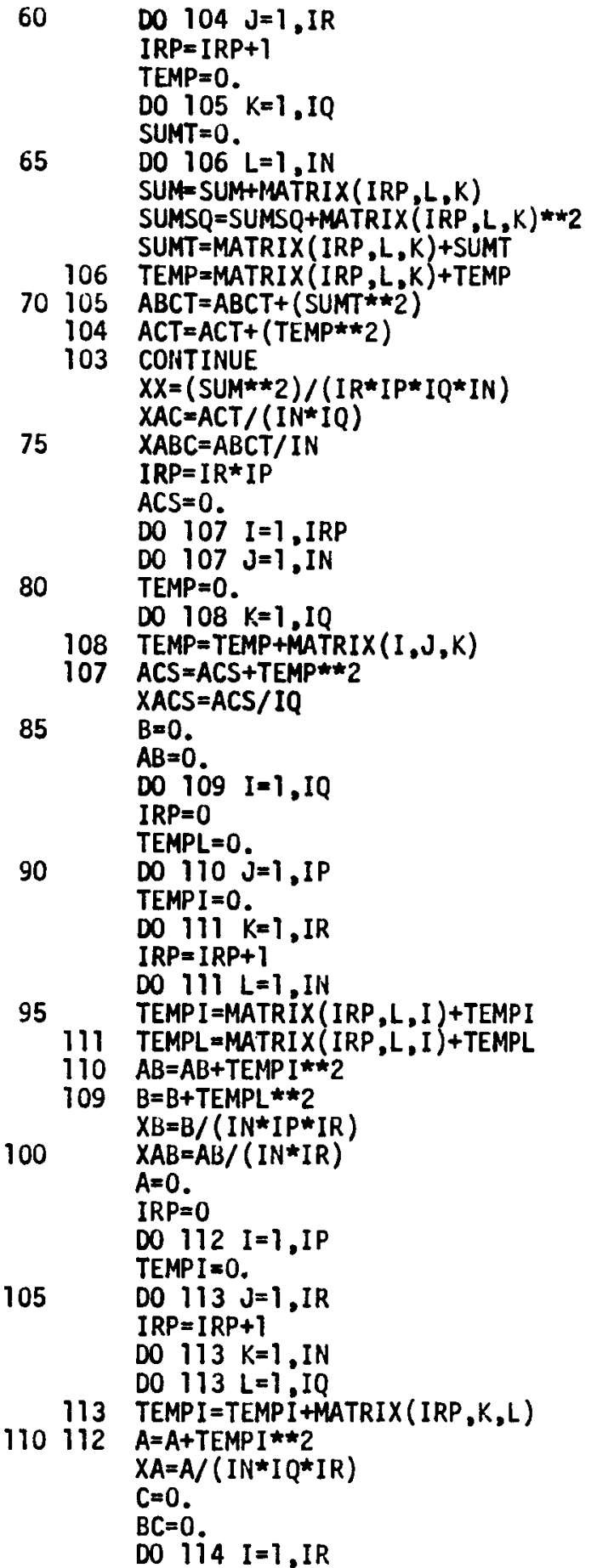

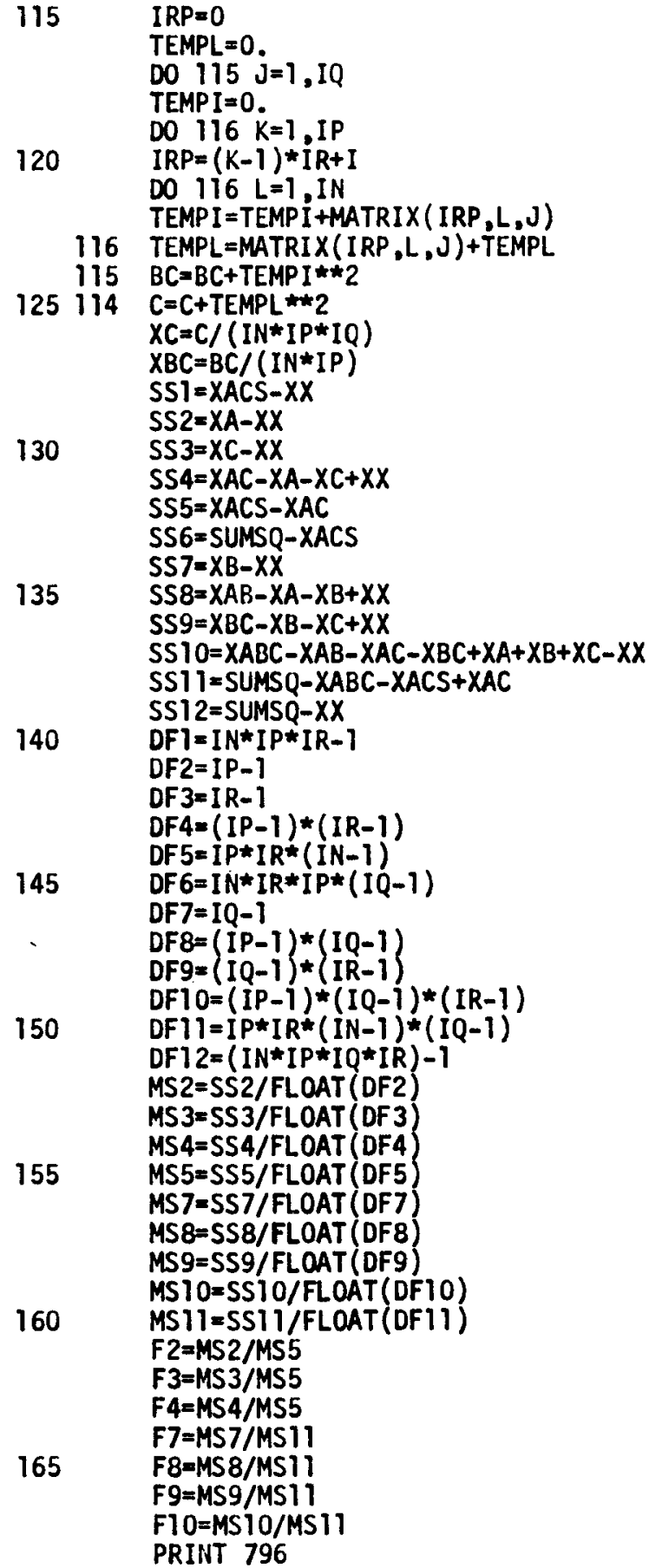

\footnotetext{
170 PRINT 67

67 FORMAT (1HO ,7X ,6HSOURCE, 12X,2HSS, 13X, 2HDF, 13X, 2HMS, 13X, 1HF) PRINT 55, SSI,DFI

55 FORMAT (1 HO, IHI , 5X, 7HBETWEEN, 7X, F10.4,9X, I2) PRINT 56,SS2, DF2, MS2,F2

17556 FORMAT (iH, IH2, 8X,1HA, 10X, F10,4,9X, 12,9X,2(F10,4,5X)) PRINT 57, SS3,DF3,MS3,F3

57 FORMAT (IH , TH $3,8 X$, IHC , 10X, F10.4,9X,I2,9X,2(F10.4, 5X)) PRINT 58, SS 4, DF 4 , MS4, F4

58 FORMAT $(1 H, 1 H 4,8 X, 2 H A C, 9 X, F 10.4,9 X, I 2,9 X, 2(F 10.4,5 X))$
} 


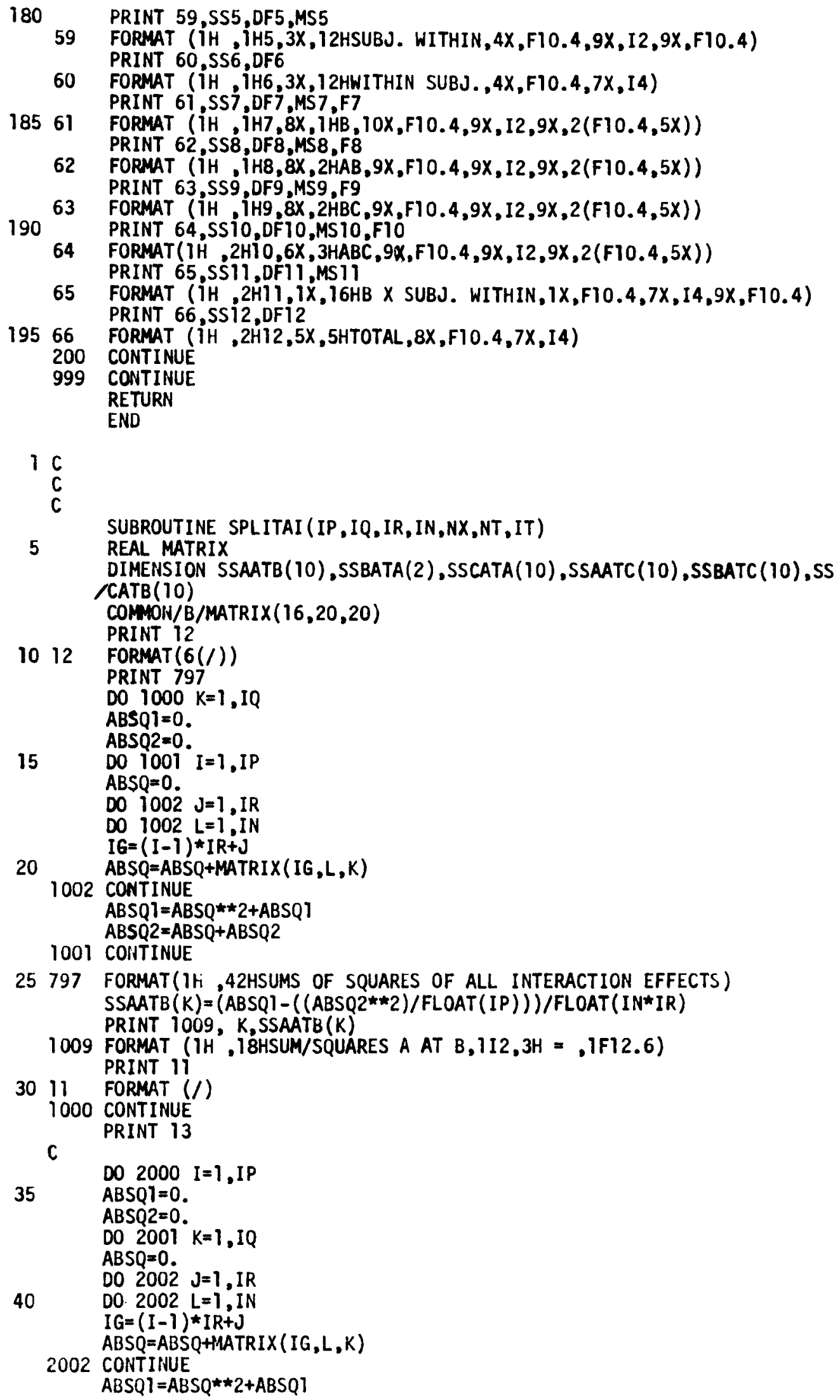




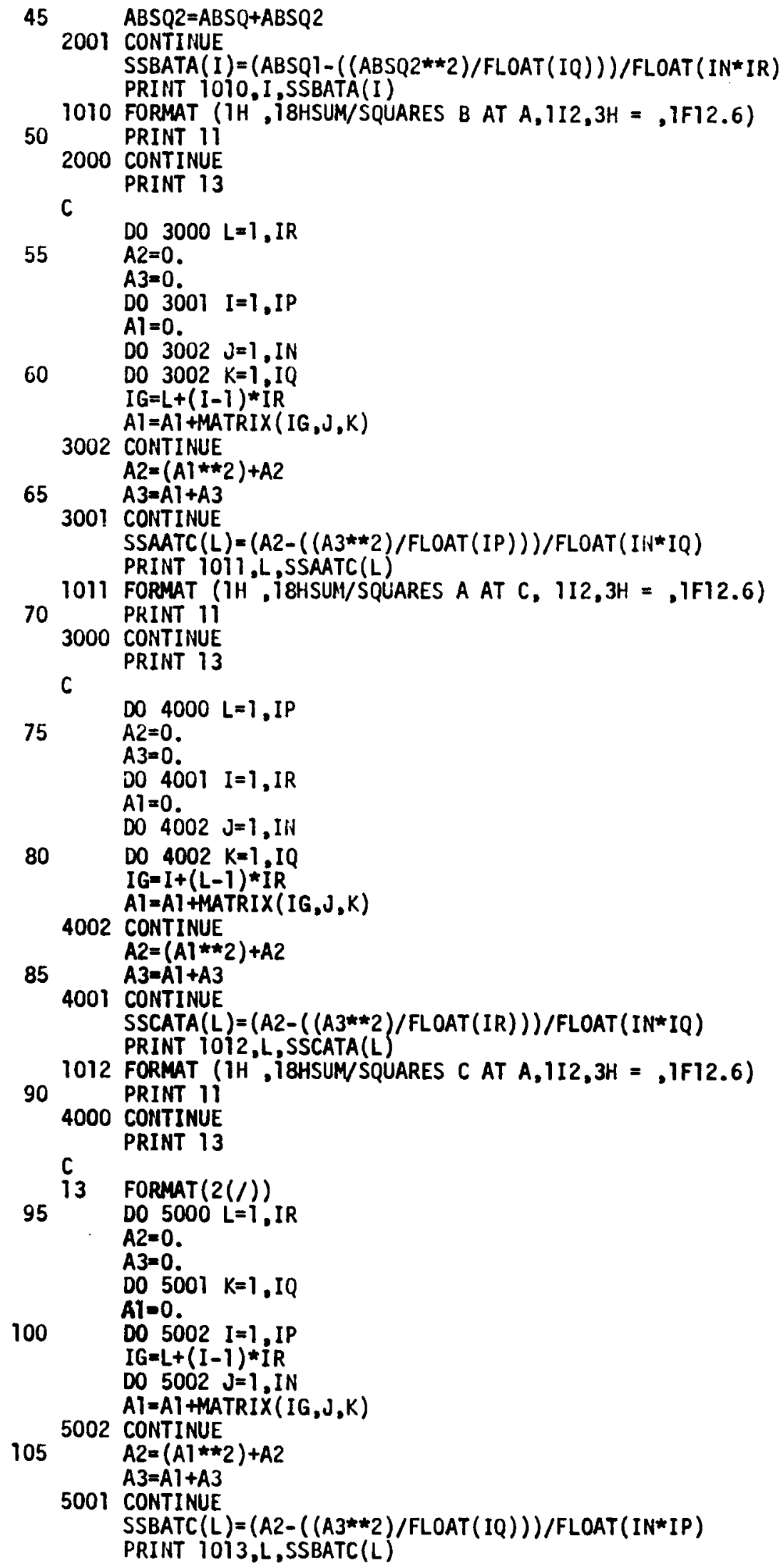




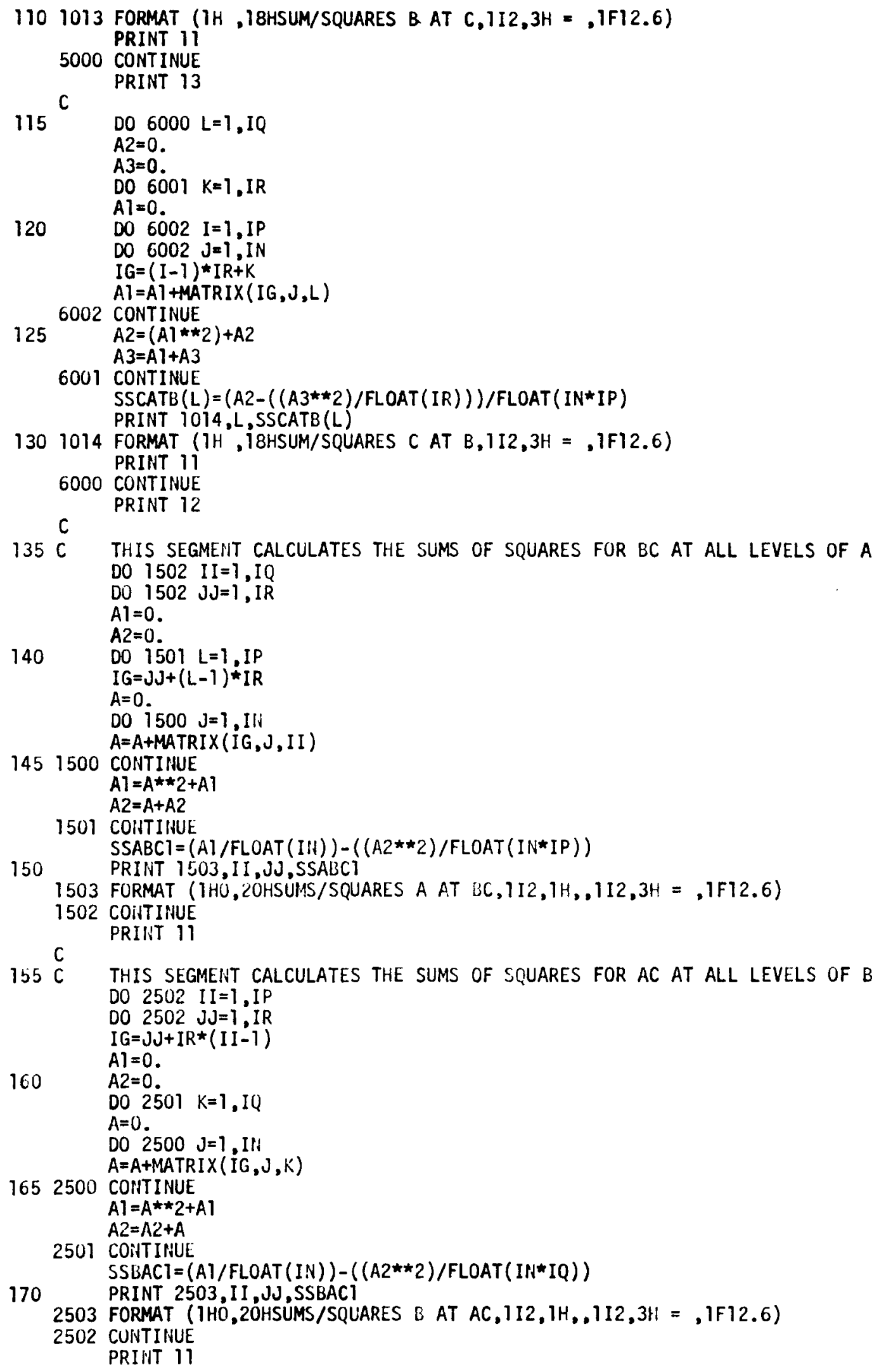

$155 \mathrm{C}$ THIS SEGMENT CALCULATES THE SUMS OF SQUARES FOR AC AT ALL LEVELS OF B DO 2502 II $=1$, IP 
C

$175 \mathrm{C}$ THIS SEGMLNT CALCULATES THE SUMS OF SQUARES FOR AB AT ALL LEVELS OF $C$ DO 3502 I $I=1$, IP

DO $3502 \quad J J=1$, IQ

$A]=0$.

$A 2=0$.

$180 \quad$ DO $3501 \quad I=1, I R$

$A=0$.

DO $3500 \mathrm{~J}=1$, Ii

$I G=1+(I I-1) * I R$

$A=A+M A T R I X(I G, J, J J)$

1853500 CONTINUE

$A l=A \star \star 2+A l$

$A 2=A+A 2$

3501 CONTIHUE

SSCABI $=(A 1 / F L C A T(I N))-((A 2 * \star 2) / F L O A T(I H * I R))$

190 PRINT 3503,11 , JJ, SSCABI

3503 FORMAT (1HO,2OHSUMS/SQUARES C AT AB, 112,1H, , 112,3H = ,IF12.6)

3502 CONTINUE

PRINT 11

$195 \stackrel{C}{C}$ THIS SEGMENT CALCULATES THE SUMS OF SQUARES FOR $C$ AT ALL LEVELS OF AB DO 4505 II $=1$, IR

$A]=0$.

$A 2=0$.

$200 \quad I G=I I+(I-1) \neq I R$

$I G=I I+(I-1) \star I R$
$D 04501 \quad K=1, I Q$

$A=0$.

DO $4500 \mathrm{~J}=1$, IN

2054500 CONTINUE

$A=A+M A T R I X(I G, J, K)$

$A]=(A \star \star 2)+A 1$

$A 2=A+A 2$

4501 CONTINUE

4502 CONTINUE

210 SABACI $=($ AI $/$ FLOAT (IN $))-((A 2 \star \star 2) / F L O A T(I N * I P \star I Q))-S S A A T C(I I)-S S B A T C($

III)

PRINT 4503, II, SABACI

4503 FORMAT (1HO,2OHSUMS/SQUARES AB AT $C, 112,3 \mathrm{H}=, 3 \mathrm{X}, 1 \mathrm{~F} 12.6$ )

4504 CONTINUE

215

C THIS SEGMENT CALCULATES THE SUAS OF SQUARES FOR B AT ALL LEVELS OF AC DO 5504 II $=1$; IQ

$220 \quad A 2=0$

DO $5502 \quad I=1, I P$

DO $5501 \quad L=1, I R$

$I G=L+(I-1) \star I R$

$225 \quad$ DO $5500 \mathrm{~J}=1, \mathrm{IN}$

$A=A+M A T R I X(I G, J, I I)$

5500 CONTINUE

$A \mid=A 1+(A \star \star 2)$

$A 2=A 2+A$

2305501 CONTINUE

5502 CONTINUE

SACAB I $=($ AI $/$ FLOAT (IN $))-(($ A2**2) $/$ FLOAT (IN*IP*IR $))-S S A A T B(I I)-S S C A T B($

/II)

PRINT 5503, II , SACAB I

2355503 FORMAT (1HO,2OHSUMS/SQUARES AC AT B, 1 I2, $3 \mathrm{H}=, 3 \mathrm{X}, 1 \mathrm{~F} 12.6)$

5504 CONTINUE

PRINT 11 


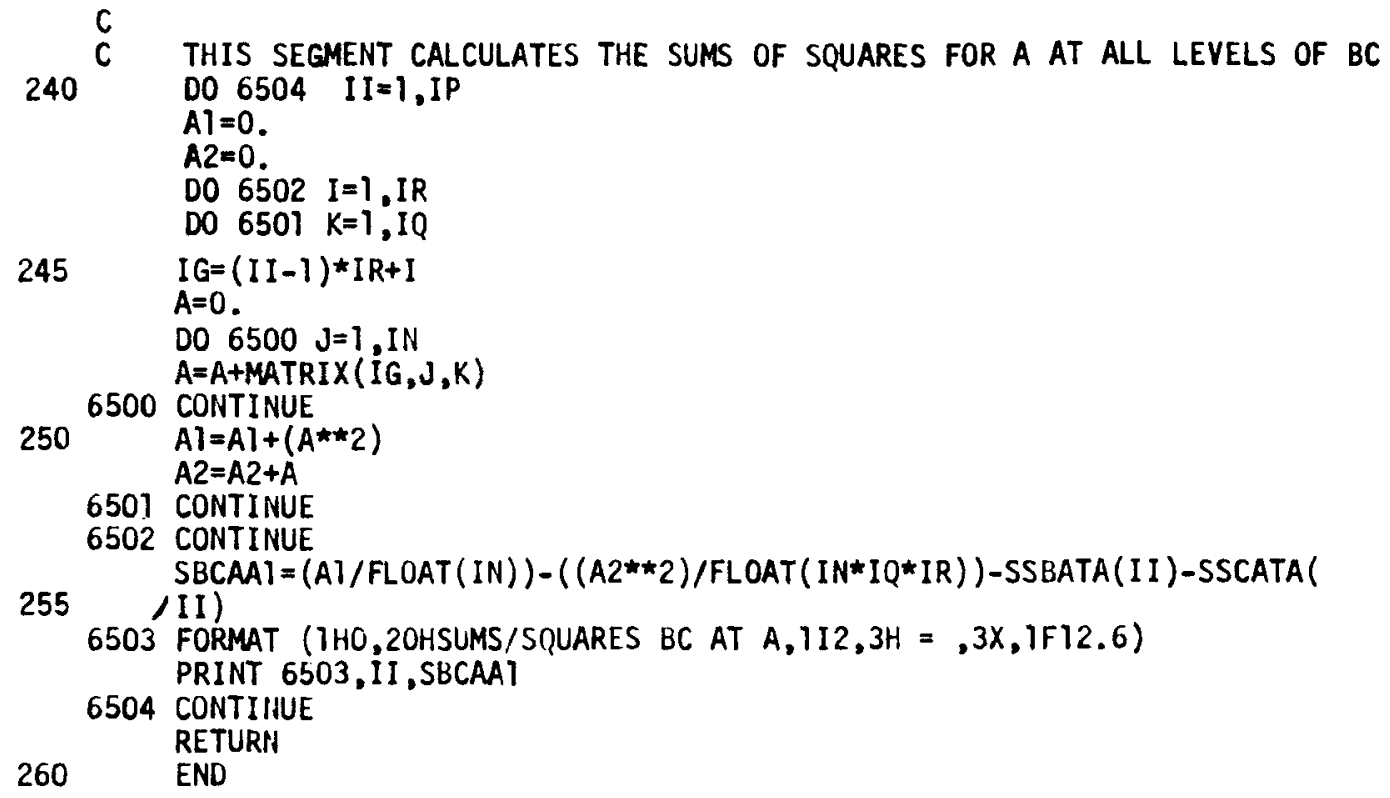

C

C THIS SEGMENT CALCULATES THE SUMS OF SQUARES FOR A AT ALL LEVELS OF BC 240 DO $6504 \quad I I=1$, IP

$A 1=0$.

$A 2=0$.

DO 6502 I $=1, I R$

DO $6501 \quad K=1,10$

$245 \quad I G=(I I-1) * I R+I$

$A=0$.

DO $6500 \mathrm{~J}=1$, IN

$A=A+M A T R I X(I G, J, K)$

6500 CONTINUE

$250 \quad A I=A I+(A \star 2)$

$A 2=A 2+A$

6501 CONTINUE

6502 CONTINUE

255 SBCAA] $=($ AI/FLOAT (IN $))-(($ A2**2) $/$ FLOAT $(I N * I Q * I R))-S S B A T A(I I)-S S C A T A($

6503 FORMAT (1HO,20HSUMS/SQUARES BC AT A,112,3H = ,3X, IF12.6)

6504 CONTIIIUE

RETURN

260 END 